\title{
Eine modale Theorie des Vertrauens
}

\author{
Zwischen wissenschaftlicher Erforschung, philosophischer \\ Analyse und religiöser Orientierung
}

Johannes Corrodi Katzenstein

\section{Einführung}

Dieser Aufsatz ${ }^{1}$ fragt nach dem prinzipiellen Verhältnis von empirischen, historischen, philosophischen und theologischen Zugangsweisen in der Erarbeitung eines nicht-reduktiven Vertrauensbegriffes. Da jede dieser unterschiedlichen Zugangsweisen in der aktuellen Vertrauensforschung eine nicht zu übersehende Rolle spielt, kommt der Frage nach den Grenzen und dem Verhältnis dieser verschiedenen Zugangsweisen entsprechende Bedeutung zu.

Zunächst stellt sich hier aber die Frage: Warum ein Begriff von Vertrauen? Ist Vertrauen nicht viel mehr als sich jemals in Begriffen, Kategorien oder theoretischen Modellen fassen lässt? Oder schärfer: Geht das Eigentliche am Vertrauen nicht verloren, wenn es logisch gefasst und begrifflich objektiviert werden soll? ${ }^{2}$

Nun ist kaum zu bestreiten, dass wir alle wissen, was Vertrauen ist - und zwar lange, bevor sich jemandem, wenn überhaupt jemals, die Frage aufdrängt, worin denn Vertrauen swirklich bestehe, oder was mit Vertrauen >eigentlich gemeint sei. Das gilt wohl ebenso für Misstrauen und den Verlust von Vertrauen. In dieser Hinsicht verhält es sich mit >Vertrauensphänomenen` nicht anders als mit unzähligen anderen Phänomenen des menschlichen Lebens. Ein vertrauter Umgang mit Göttern, Menschen, Gemeinschaften, Naturprozessen

\footnotetext{
1 Dieser Aufsatz ist entstanden im Zusammenhang des interdisziplinären Forschungsprojekts , Vertrauen verstehen Mercator Schweiz und dem Schweizerischen Nationalfonds (SNF) finanziell getragen wurde. Diesen sei an dieser Stelle herzlich gedankt.

2 Wo z.B. empathisches oder liebendes Vertrauen zwischen >Ich und Du< (Martin Buber) als Richtschnur für das Verständnis von eigentlichem Vertrauen genommen wird, ist eine solch missbilligende Haltung gegenüber Begriffen und Theorien meist nicht weit zu suchen. So erklärt Joseph Godfrey: "Buber's ontology [...] is not one to be captured in categories. "Vgl. Joseph Godfrey, Trust of People, Words, and God: A Route for Philosophy of Religion, Notre Dame 2012, 238; s. auch 229 u. Anm. 21, 438.
} 
und -dingen zeichnet das vortheoretische Leben und praktische Wissen von Menschen zu allen Zeiten aus, ebenso wie der `Fall aus diesem alltäglichen Vertrauen heraus.

Ein solch elementares Wissen der begegnenden Lebenswirklichkeit, wie auch dessen ständige Bedrohung, ist noch nicht einmal auf Menschen beschränkt. Das >Wissen der jungen Katze um die Muttermilch kann als Illustration dafür dienen. Darüber hinaus eröffnet der menschliche Spracherwerb nun zwar eine neue (Um)Welt, in der instinktgeleitetes Wissen nicht genügt, um sich verlässlich orientieren zu können. Aber auch diese sprachlich-symbolisch vermittelte Welt besteht zunächst - und später immer auch - aus konkreten Dingen, Ereignissen, Gemeinschaften, Institutionen, etc. Es geht mir im Folgenden also keineswegs darum, begrifflich-theoretisch erhobenes Wissen als vermeintliche Alternative zum konkreten Umgang mit lebensweltlicher Erfahrungswirklichkeit aufzubauen. Konkretes Wissen-Wie ist immer praktisches Wissen, das in allen möglichen gesellschaftlichen Bereichen, und so auch im Bereich der Wissenschaft, einschliesslich Philosophie und Theologie, eine unverzichtbare Rolle spielt. Es kann durch theoretisch-begriffliches Wissen-Dass niemals prinzipiell `abgelöst oder `ersetzt $\iota$ werden.

Andersherum stellt sich dann aber auch die Frage: Was soll Wissenschaft, Philosophie und Theologie im Gegensatz zu Kunst, Religion und anderen praktischen Erfahrungs- und Gestaltungsweisen von gelebter Wirklichkeit auszeichnen, wenn nicht ein theoretischanalytischer Zugang, der sich in Begriffen, Modellen und Theorien niederschlägt? Beobachtung und Reflexion gibt es auch auf der Stufe des vortheoretischen Lebensvollzugs. Dieser bewährt sich nicht erst aus der theoretisch-wissenschaftlichen Distanz einer begrifflich-systematischen Analyse alltäglicher Beobachtungen und Reflexionen. Das zu glauben, wäre etwas zu viel des rationalistischen Vernunftvertrauens.

Im weiteren gehe ich davon aus, dass jede naturwissenschaftlichempirische, historische, philosophische und theologische Erforschung von Vertrauen unweigerlich theoretisch-begrifflichen Charakter hat oder haben sollte. Wer in einem wissenschaftlichen Kontext Vertrauen oder irgendetwas beschreibt, beschreibt implizit oder explizit immer auch ein theoretisch-begriffliches framework. Aber wie gesagt, das sei hier nochmals ausdrücklich unterstrichen, verbindet sich damit keineswegs die vermessene Behauptung, dass theoretischbegriffliches Wissen-Dass eine Art höheres Wissen darstellt, das Alltagserfahrung, Kunst, Religion, etc. prinzipiell hinter sich zurücklassen könnte. Praktisches Wissen kann theoretisch-begrifflich 
'vertieft und systematisiert, aber nicht überboten werden. Auch die Chemikerin geniesst den Wein und nicht eine Synthese unterschiedener Substanzen.

Nun gibt es nicht die Wissenschaft des Vertrauens. Soviel scheint evident. Es ist auch schwer einzusehen, warum die theoretische Beschäftigung mit Vertrauen z.B. mehr mit Philosophie oder mit Theologie zu tun hat als mit empirischen oder historischen Disziplinen. Das Umgekehrte gilt natürlich ebenso.Vertrauen hat, wie alles, worüber sich sprechen und forschen lässt, viele Aspekte. Es kann in unterschiedlichen Lebenssituationen, historischen Epochen, Wissensdisziplinen und spirituellen Traditionen sehr verschiedene Dinge bedeuten. Der konkreten Erscheinungsformen von Vertrauen gibt es faktisch immer mehr als man beschreiben und analysieren könnte. Und zugleich gibt es immer auch verschiedene Beschreibungsebenen und theoretisch-begriffliche Zugangsweisen. In beiderlei Hinsicht unterscheidet sich Vertrauen nicht von anderen Dingen. Es gibt nicht das Vertrauen, sowenig wie es die Liebe oder die Gerechtigkeit gibt. Wir leben nun mal in einer pluralen Wirklichkeit.

Das mag trivial erscheinen. Doch tritt bereits hier die erste Komplikation auf. Die Geschichte des westlichen Forschens und Denkens ist seit dessen greifbaren Anfängen nämlich von einer bestimmten Gesinnung geprägt, der gemäss $\mathrm{x}$ >eigentlich y ist. Die Vielgestaltigkeit der Phänomene liegt so gesehen ১bloss` auf der Ebene der Erscheinungen - sin Wirklichkeit ‘, oder sobjektiv ‘, sind die Dinge ganz anders als sie uns ssubjektiv erscheinen mögen. Diese tiefliegende Gesinnung hat mit spezifischen Vertrauensphänomenen noch nichts zu tun. Denn es gibt tatsächlich nichts in unserer Welt, vor der eine solche Grundeinstellung halt machen müsste. Und oft führt sie zu einer bestimmten Art wissenschaftlicher Theoriebildung, der gemäss $\mathrm{x}$ letztlich ınichts anderes als y ist. Das ist die verbreitete Sprache des theoretischen Reduktionismus. Ob dieser Reduktionismus nun 'wissenschaftlich', d.h. wissenschaftlich unverzichtbar ist, wie man vielleicht annehmen könnte, ist wissenschaftstheoretisch aber längst nicht ausgemacht.

Freilich hat auch der theoretische Reduktionismus viele Gesichter, darunter freundliche und weniger freundliche. Hier stehen die eher unfreundlichen imVordergrund. DerWissenschaftsphilosoph P. Feyerabend hat den Versuch unternommen, entscheidende Momente in einer historischen und systematischen Entwicklung des Bewusstseins herauszuarbeiten, die er die Vernichtung der Vielfalt nennt. In der Vorrede seines gleichnamigen, unvollendet gebliebenen Buches schreibt er: "Eine der wichtigsten Folgen dieser >Vernichtung der 
Vielfaltı, dieser Machtergreifung kruder und monolithischer Ideen, ist die triste Welt, in der einige von uns heute leben, eine Welt, die nur wissenschaftlichen Machtsprüchen und ökonomischen Anordnungen gehorcht. $\mathbb{1}^{3}$

In dieser tristen Welt sollen demnach allein natürliche und soziale Gesetze herrschen, deren Wesen und unumstössliche Geltung wissenschaftlich-objektiv festgestellt werden kann. Feyerabend hat hier eine ähnliche Situation im Blick wie der politische Philosoph Eric Voegelin, der die Grundhaltung eines Gründervaters des wissenschaftlichen Positivismus des neunzehnten Jahrhunderts, Auguste Compte, folgendermassen auf den Punkt brachte: "Der Horizont des Menschen ist von einer festen Mauer aus Fakten und Gesetzen der Phänomene umgeben. ${ }^{4}$ Dieses >Vorurteil bezüglich Mensch und Welt kann wohl als paradigmatisch für jedes moderne swissenschaftliche bzw. szientistische Immanenzdenken bezeichnet werden.

Die unbeantwortete Frage ist hier dann bloss noch die, wie die subjektiven Lebenseinstellungen - Vorstellungen, Wünsche, Hoffnungen, etc. - einer scheinbar unheilbar religiös gesinnten Masse in rationalen Einklang mit der so (miss)verstandenen Welt und der durch sie festgelegten menschlichen Natur gebracht werden können. ${ }^{5}$ Das ist eine Frage an den im Geist von Aufklärung und Vernunft herrschenden Erzieher und Gesetzgeber. Aber wie auch immer sie jeweils beantwortet wird: An der harten Wirklichkeit bzw. der objektiven Geltung der wissenschaftlich-objektiv erhobenen Fakten und Gesetzmässigkeiten selbst gibt es scheinbar nichts mehr zu rütteln.

Auf dem Hintergrund einer verwissenschaftlichten und ökonomisierten Lebenswirklichkeit, wie sie von Feyerabend, Voegelin und vielen anderen analysiert wird, ${ }^{6}$ kann die Frage nach der Möglichkeit eines nicht-reduktiven Vertrauensbegriffes deshalb kaum als selbstverständlich gelten.Vertrauen ist dann im Kern

\footnotetext{
3 Paul K. Feyerabend, Die Vernichtung der Vielfalt: Ein Bericht, Wien 2004. Der Titel der Originalausgabe lautet: The Conquest of Abundance.

4 Eric Voegelin, Die Krise. Zur Pathologie des modernen Geistes, Paderborn 2007, 237.

5 Nicholas Wolterstorff, Inquiring about God, Cambridge NY 2010, 22.

${ }^{6}$ Ein locus classicus dieses Typus von Gesellschaftsdiagnose ist Max Horkheimer/ Theodor Adorno, Dialektik der Aufklärung. Philosophische Fragmente, Frankfurt a.M. ${ }^{3} 2003$. Vgl. auch Jürgen Habermas, Faktizität und Geltung. Beiträge zur Diskurstheorie des Rechts und des demokratischen Rechtsstaats, Frankfurt a.M. ${ }^{1} 1992$, 64. Eine subtile theologiegeschichtliche Herleitung der modernen Gestalt von ökonomischer Herrschaft bietet Giorgio Agamben, Herrschaft und Herrlichkeit. Zur theologischen Genealogie von Ökonomie und Regierung, Frankfurt a.M. 2010.
} 
vielleicht nur das, was die sharten (Wissensdisziplinen mit ihren Methoden (gegenwärtig wohl vor allem die Neurowissenschaften) an diesem vielgestaltigen Phänomen erfassen und erklären können. Andere gesellschaftliche Leistungsbereiche wie Politik und Erziehung, die mit der Gestaltung subjektiver Faktoren zu tun haben, können und sollen auf diesem Wissen aufbauen und es gesellschaftlich nutzbringend einsetzen. Vertrauen gerät dann in den `öffentlichen ‘ Blick als unverzichtbare Bedingung des ökonomischen Wachstums und als Ressource des sozialen Zusammenhalts. ${ }^{7}$ Besonders in Zeiten des verlangsamten ökonomischen Wachstums oder einer drohenden Wirtschaftskrise drängt sich die Frage der Erneuerbarkeit der Ressource Vertrauen in den medialen und gesellschaftspolitischen Vordergrund.

Natürlich ist die Geschichte an diesem Punkt nicht zu Ende. Gegen die eben skizzierte Betrachtungsweise erhebt sich auch Widerstand. Es scheint, dass je mehr ein reduktionistischer, oder, oft scheinbar synonym verwendet, ein begrifflich-aussagender ('prädikativer $)$ Zugriff auf die Phänomene die theoretische Oberhand gewinnt, desto massiver sich bei vielen Denkern eine existential-phänomenologische Reaktion dagegen einstellen will, deren Wurzeln ebenso im neunzehnten Jahrhundert liegen wie die des wissenschaftlichen Positivismus bzw. Szientismus. Man kann dann entsprechend versucht sein, die radikale Offenheit gegenüber den Dingen des Lebens mit einem nicht-theoretischen Denken in Verbindung bringen zu wollen, das sich mir allein in und durch den eigenen Daseinsvollzug erschliesst. Der Rückgewinn von rauthentischem Vertrauen in die Dinge diesseits ihrer logisch-objektivierenden Vereinnahmung in der Beobachter- und Organisationsperspektive drängt somit in den Mittelpunkt des philosophischen und theologischen Interesses.

Nun ergibt sich hier aber eine neue Schwierigkeit. Die Frage drängt sich auf, ob eine vorprädikative Zugangsweise zu den Phänomenen nicht in eine antitheoretisch-theoretische Reduktion dieser Phänomene umschlagen kann, die auf ihre Weise nicht weniger verkürzend wirkt als der skizzierte theoretische Reduktionismus. Die leitende Unterscheidung ist hier zwar nicht mehr die zwischen blosser Erscheinung und wirklicher Realität. Sie verläuft vielmehr

\footnotetext{
7 Spuren einer solchen Einstellung können selbst dort noch auftreten, wo die erwähnten Formen des theoretischen Reduktionismus überwunden werden sollen, aber Vertrauen als ssoziales Kapital angesprochen wird. Damit werden irreführende Konnotationen des Berechnens freigesetzt: "The irony is that the limits on calculativeness are realized by examining user-friendly terms - of which strust is one - in a thoroughly calculative way." Oliver E. Williamson, Calculativeness, Trust, and Economic Organization, in: Journal of Law and Economics 36, 1 (1993), 453-486, 486.
} 
zwischen dem, >was sich zeigt, so wie es sich von ihm selbst her zeigt (M. Heidegger) ${ }^{8}$ und den Dingen, wie sie selbst durch den vortheoretischen Alltagsverstand immer schon $<$ logisch objektiviert worden sind um deren vermeintliche Beherrschbarkeit sicherzustellen. Ihrer Intention nach soll diese Unterscheidung dem >logischen Vorurteil (D. Dahlstrom) ${ }^{9}$ entgegengetreten, das sich im westlichen Denken über die Jahrhunderte festgesetzt hat - und unser Verständnis von Wahrheit und Sein begrenzt und verdunkelt. Doch hier liegt das Problem. Weil für Heidegger in der modernen Massengesellschaft unser alltägliches Handeln und Bewusstsein der Dinge diesem , Vorurteik bereits zum Opfer gefallen ist, wird Wahrheit, wie L. Zuidervaart kritisch notiert, zum unerklärlichen Privileg authentischer Existenz. "This privileging of authentic existence ensnares his [i.e. Heidegger's] conception in the self-referential incoherence of theorizing what, according to his own theory, cannot be theorized. $«^{10}$

Das Verhältnis zwischen authentischem Vertrauen in die Dinge und dessen begrifflich-theoretischer Erschliessung bleibt hier also merkwürdig in der Schwebe. Sicher ist aber, dass wo immer sich Geisteswissenschaften, Philosophie und Theologie einseitig an einer vorprädikativen Herangehensweise an die Vertrauensthematik orientieren, sie in ein potentiell antagonistisches Verhältnis zu natur- und gesellschaftswissenschaftlichen Zugängen geraten. Phänomenologisch-hermeneutische und wissenschaftlich-theoretische Zugangsweisen sind dann bestenfalls aber so sgrundverschieden<, dass sie auf getrennten Ebenen bzw. in parallelen Diskursuniversen operieren. Begrifflich-theoretisches Erklären im Bereich der `Natur (Kausalität) versus phänomenologisch-hermeneutisches Aufzeigen und vollziehendes Verstehen im Bereich der `Freiheit< (Sinn). Dieser Dualismus von Natur und Freiheit ist, in allen seinen Ablegern und Versionen, nicht weniger Teil der intellektuellen Stimmung und des begrifflichen Repertoires des vergangenen Jahrhunderts als das eingangs von Feyerabend skizzierte Szenario eines theoretischen und ökonomistischen Reduktionismus.

Das Problematische an dieser Situation ist nun aber, dass sich die soeben unterschiedenen Zugangsweisen gleichzeitig bedingen und dennoch gegenseitig auszuschliessen scheinen. Einerseits erscheinen die verschiedenen Zugangsweisen auf ihrem Gebiet als grundsätzlich

\footnotetext{
8 Martin Heidegger, Sein und Zeit, Tübingen ${ }^{19} 2006,34$.

9 Daniel O. Dahlstrom, Das logische Vorurteil. Untersuchungen zur Wahrheitstheorie des frühen Heidegger, Wien 1994.

${ }^{10}$ Lambert Zuidervaart, Artistic Truth. Aesthetics, Discourse, and Imaginative Disclosure, Cambridge UK 2004, 78.Vgl. Dahlstrom, Das logische Vorurteil, 20.
} 
berechtigt, andererseits lässt sich das jeweilige Gebiet aber nicht von einer gemeinsamen Sache her begrenzen, ohne dass Identität und Einheit der Sache selbst zu zerfallen drohen oder diese sich dialektisch $\mathrm{zu}$ halbieren beginnt. Immer scheint die Dimension von 'Natur (Kausalität) oder die Dimension von `Freiheit` (Sinn/Intentionalität) fundamentaler für das Verständnis der Sache des Vertrauens zu sein. ${ }^{11}$ Das ist aber darum fatal, weil ohne die Unterstellung einer gemeinsam vorfindlichen Sache bzw. eines nicht-konstruierten, vortheoretischen `Erfahrungsganzen die Frage der jeweiligen Zuständigkeiten und Kompetenzen der unterschiedlichen Zugangsweisen und Disziplinen in der interdisziplinären Erforschung von Vertrauen schon gar nicht mehr gestellt, geschweige denn sinnvoll beantwortet werden kann.

\section{Vertrauen als modaler Aspekt}

Wenn nun keine aporetischen oder sonst wie unfruchtbaren Alternativen zwischen den verschiedenen Zugangsweisen zurVertrauensthematik fortgeschrieben werden sollen, muss man sich davor hüten, jede Begriffs- und Theoriebildung unter den Generalverdacht des Reduktionismus zu stellen. Ein genuin kritischer Denkansatz kann keine der unterschiedenen Zugangsweisen - Beobachter- und Vollzugsperspektive - der jeweils anderen vorordnen wollen, ohne deren inneres Verhältnis zu klären. Und dies kann nicht ohne logische Begriffsbildung bzw. ohne ein erweitertes Theorieverständnis geschehen.

Damit ist zumindest eine potentielle Schwierigkeit beiseite geräumt. Mein Interesse gilt also einem nicht-reduktiven, theoretischen Vertrauensbegriff. Dabei gehe ich von der im weiteren zu entfaltenden Annahme aus, dass verschiedene wissenschaftliche Disziplinen verschiedene Aspekte von Vertrauen erschliessen - organische, psychische, kulturelle, soziale, ökonomische, rechtliche, moralische, etc. - ohne notwendig in ein Konkurrenzverhältnis zu einander geraten zu müssen. Keiner dieser begrifflich-theoretischen Zugänge zum Thema des Vertrauens ist in der hier entfalteten Perspektive einem anderen a priori überlegen.Vielmehr hat jeder seinen

${ }^{11}$ Eine Folge dieser theoretischen Aporie zeigt sich in der periodisch wiederkehrenden Gegenüberstellung von natürlicher Kausalität und menschlicher Freiheit auf allen möglichen Gebieten, nicht nur in der wissenschaftlichen Erforschung von Vertrauen. 
eigenen Gegenstandsbereich. Die Philosophie kann helfen aufzeigen, wie dies möglich sein soll. ${ }^{12}$

Eine wichtige Vorentscheidung, die jede begrifflich-theoretische Zugangsweise zum >Phänomen ‘ des Vertrauens treffen muss, ist nun in der eben vorgenommenen Kategorisierung schon angelegt. Die Frage stellt sich: Ist Vertrauen ein Phänomen, d.h. ein konkretes Etwas sunter einer bestimmten Symbolisierung oder Beschreibung? Oder ist der Ausdruck /Vertrauen` mit dem Ausdruck `Leben` vergleichbar, der, trotz einer oft irreführenden Gebrauchsweise, gerade nicht auf ein konkretes Etwas - auf ein bestimmtes Lebewesen, ein Ereignis, ein Prozess, oder eine irgendwie geartete Kraft - verweist, sondern vielmehr eine grundlegende Dimension der menschlichen Erfahrungswirklichkeit im Blick hat, an der jedes Lebewesen teilhat? Im zuletzt genannten Fall würde sich ein konkreter Vertrauensakt oder eine Vertrauensbeziehung zu >dem 〈Vertrauen analog verhalten wie ein konkretes Lebewesen oder ein biotischer Prozess zu >dem Leben $^{13}$ - oder wie eine räumliche Figur zum Raum. Es genügt, sich die Frage auf diese Weise zu stellen, damit das hier anstehende Problem deutlich wird. Das Problem ist, wie seit der so genannten slinguistischen Wender im Denken des zwanzigsten Jahrhundert oftmals festgestellt wurde, dass unsere alltägliche Sprache das begrifflichtheoretische Denken förmlich dazu einlädt, aus substantivischen Ausdrücken wie >Raum ihrerseits (quasi-)raum-zeitliche Entitäten, Ereignisse oder Prozesse zu bilden. Doch freilich ist das Leben weder lebendig noch der Raum räumlich ausgedehnt; und das Vertrauen ist im Gegensatz zu konkreten menschlichen Beziehungen und Institutionen weder von realem noch imaginiertem Vertrauensschwund bedroht.

Obwohl ich den substantivischen Ausdruck im Folgenden weiterhin verwende, soll Vertrauen hier nun anders als substantivisch gedacht werden. Vertrauen erscheint dann nicht als ein bestimmtes Was, sondern als ein bestimmtes Wie der menschlichen Erfahrungswirklichkeit und des menschlichen Lebensvollzugs. Mit H. Dooyeweerd gesprochen ist Vertrauen ein modaler Aspekt, ein Modus des Erfahrens und Gestaltens von Lebenswirklichkeit, der unseren Lebensvollzug in seiner Gesamtheit durchzieht. Anders gesagt: Nicht

\footnotetext{
12 Die beiden Denker, auf die ich mich in meinen Überlegungen massgeblich abstütze, sind Herman Dooyeweerd (1894-1977) und Eric Voegelin (1900-1985). Diese haben zwar keine eigene Vertrauenstheorie vorgelegt, oder zumindest nicht unter diesem Titel. Dooyeweerd war von Haus aus Rechtstheoretiker und Voegelin der politischen Philosophie zugetan.

13 "Life is a fundamental modality, not a concrete phenomenon." Herman Dooyeweerd, A New Critique of Theoretical Thought, Lewiston 1997, 2, 108.
} 
jedes Phänomen in unserer Erfahrung gehört zur Menge der Phänomene, die unter die Bezeichnung ,Vertrauen fallen. Aber jedes Phänomen in unserem Lebenshorizont kann unter dem Aspekt des Vertrauens und der Vertrauenswürdigkeit analysiert und erforscht werden. So verstanden kann Vertrauen freilich dann auch wieder als Phänomen bezeichnet werden. Es zeigt sich, tritt auf, manifestiert sich oder erscheint (Gr. phainomenon) an allen Dingen, wirklichen und möglichen, vergangenen und zukünftigen.

Aber hier liegt der entscheidende Unterschied: Anders als eine konkrete Sinnes- oder Zeiterscheinung, die auch anders hätte ausfallen, oder gar nicht erst auftreten können (etwa die nächste Finanzkrise), macht Vertrauen im modalen Sinn soziale Phänomene wie Lehrbücher, Kredite, Verträge, Freundschaften, Liebesbeziehungen, demokratische Regierungen, Freiheitsrechte, religiöse Dogmen, etc. allererst möglich. Dasselbe gilt dann ebenso für den Missbrauch der aufgezählten Dinge, der oft in Misstrauen gründet oder mündet. Wer also von Vertrauen oder Misstrauen spricht - welches konkrete Etwas damit auch jeweils gemeint sein mag - bezieht sich immer auch auf eine universale und notwendige (= transzendentale) Ermöglichungsbedingung von gelingendem Leben. Denn was gibt es im menschlichen Lebensvollzug, das nicht irgendwie vertrauensrelevant werden könnte? Was in dieser Welt könnte nicht zum ১Ort< werden, wo sich Vertrauen thematisieren liesse?

Vertrauen ist also kein Phänomen unter Phänomenen, sondern mit allen konkreten Phänomenen gegeben.Vertrauen ist dann nicht primär ein sortaler Begriff, mit dem etwas identifiziert bzw. als etwas beschrieben und unterschieden wird (`Blues`, >Umweltschutz`), sondern es macht >Vertrauensphänomener allererst möglich - ähnlich wie `Leben das Auftreten biotischer Entitäten und Prozesse, oder 'Raum das Auftreten geometrischer Formen bzw. Operationen möglich macht. Konkrete Vertrauensbeziehungen, Vertrauensakte, Vertrauensprozesse und dergleichen partizipieren in modaler Sichtweise am Vertrauen als ihrem nicht-konkreten (nicht-empirischen oder -historischen) Ermöglichungsgrund.

Zwei Punkte möchte ich an dieser Stelle festhalten. Erstens: Es gibt nichts und niemanden innerhalb des menschlichen Erfahrungshorizonts, der von der Frage nach dessen Verlässlichkeit bzw.Vertrauenswürdigkeit ausgeschlossen wäre - und zwar nicht deswegen, weil etwas von dieser Art noch nicht empirisch entdeckt oder historisch analysiert worden wäre. Zweitens: Wenn alle empirisch und historisch unterscheidbaren Vertrauensphänomene an einem bestimmten Modus der menschlichen Erfahrung und des Lebensvollzugs teil- 
haben, der hier als ,Vertrauen e eingeführt wurde, kann keines dieser Phänomene zum Vertrauen im essentiellen oder eigentlichen Sinn werden. Keines ist Sach- oder Verstehensgrund aller anderen. Die Lozierung des 'wirklichen Vertrauens in einer bestimmten Erscheinungsform davon wird somit obsolet. Zum Beispiel: Ist Vertrauen in den Verhandlungspartner in ökonomischen Tauschsituationen fundamentaler als Ich-Du-Vertrauen zwischen Liebespartnern, oder verhalten sich die Dinge nicht vielmehr umgekehrt? Es gibt Annahme zum Verdacht, dass auf dieser Ebene jede Antwort gleichermassen arbiträr bleiben muss, wenn nicht zuerst der modale Sinn von Vertrauen geklärt wird.

\section{Die Vorgängigkeit von Vertrauen}

Nichts kann sich dem Vertrauensthema entziehen, wenn Vertrauen ein modaler Aspekt menschlicher Existenz ist. Die hier entfaltete Denkweise hat ihre Wurzeln in einer Intuition, die man die Intuition der Vorgängigkeit von Vertrauen nennen könnte. Was damit gemeint ist, zeigt sich an unzähligen verschiedenen Beispielen. Etwa hier: Wer anderen misstraut, muss seinem eigenen Misstrauen und seinen Verdachtsmomenten gegenüber x vertrauen (können). Misstrauen und Verdacht spielen in der Arbeit des Detektivs und des Strafverfolgers eine zentrale Rolle. Wie J. Godfrey bemerkt: »Suspicion can be a craftbound excellence: for an investigator, suspicion is a virtue. Thus, it is not only trust that nests in an environment of trust; suspicion can nest in trust. ${ }^{14}$ Vertrauen und Misstrauen können somit nicht einfach als sich wechselseitig ausschliessende Phänomene bestimmt werden.

Ein anderes, in unserem Zusammenhang näherliegendes Beispiel: In jedem theoretischen Akt und in jedem philosophischen oder wissenschaftlichen Versuch, Vertrauen begrifflich zu analysieren, modellieren oder objektivieren, wird Vertrauen simmer schon in Anspruch genommen. Dies ist nicht bloss darum der Fall, weil jede philosophische Reflexions- und wissenschaftliche Forschungspraxis einen sozialen Aspekt hat. Theoretisches Denken und Verstehen lebt zuinnerst von Vertrauen. Denn erstens gäbe es ohne psychischsensitives Vertrauen in unsere Wahrnehmung keine menschliche Erfahrungswirklichkeit, die reflexiv erschlossen und theoretischempirisch erforscht werden könnte; und zweitens können wir ab einer bestimmten mentalen Entwicklungsstufe nicht umhin, uns der Erschliessungskraft von Symbolen, Begriffen und theoretischen Mo-

\footnotetext{
${ }^{14}$ Godfrey, Trust of People, Words, and God, 155.
} 
dellen (bzw. der Argumente, die sich darauf stützen) anzuvertrauen. Ohne Begriffsvertrauen gibt es keine kognitive Orientierung und keine Möglichkeit der theoretisch-diskursiven Wahrheitsfindung.

Der Bezug zu der eingangs erwähnten Gesellschaftsdiagnose Feyerabends liegt auf der Hand. Wer sich der vermeintlichen Unhintergehbarkeit eines szientistischen Zugriffs auf die Vertrauensthematik entziehen will, kann auf den Gedanken kommen, dass sich selbst und gerade in einer solchen Zugangsweise eine spezifisch westliche Form von Vertrauen versteckt hält. Summarisch könnte man dieses Vertrauen als ein technisch-wissenschaftliches Weltvertrauen bzw. als Vertrauen in ein swissenschaftliches Weltbild bezeichnen. Dieses Wissenschaftsvertrauen ist unter modernen Gesellschaftsbedingungen eng mit anderen Formen von Vertrauen, etwa mit einem ausgreifenden Technik- und Marktvertrauen verbunden. Ohne die ihnen entsprechenden Formen von Vertrauen lässt sich die zentrale Bedeutung der angesprochenen Leistungssphären der modernen Gesellschaft kaum erklären - selbst wenn vielleicht erst ein realer oder imaginierter Vertrauensschwund diese Bedeutung in den Vordergrund unserer Aufmerksamkeit drängt. Es liegt also im Bereich des wissenschaftlichen Interesses selbst, das moderne Vertrauen in die 'wissenschaftliche Vernunft $<$ und ein darauf aufbauendes Technikund Marktvertrauen zu erhellen.Vertrauen kann dann freilich - das ist die zentrale These - nicht bloss als ein zu erforschendes Phänomen neben anderen in den Blick kommen. Vielmehr stellt Vertrauen als irreduzibler Modus der Erfahrungswirklichkeit zugleich eine konstitutive Ermöglichungsbedingung der einzelwissenschaftlichen Erforschung konkreterVertrauensphänomene selbst dar. Der Anschluss wissenschaftlicher Fragestellungen an transzendental-philosophische Fragestellungen ergibt sich so gesehen völlig zwanglos. ${ }^{15}$

Vertrauen wird nun nicht nur in Anspruch genommen, sbevor es wissenschaftlich erforscht werden kann, es wird immer auch auf ganz bestimmte Weise in Anspruch genommen. Dieser Punkt wäre vielleicht kaum hervorhebenswert, wenn in der westlichen

${ }^{15}$ Da gegenwärtig die intellektuelle Stimmung einer transzendentalphilosophischen Auffassung von Philosophie oft entgegensteht, ist es vielleicht nicht unnütz hier anzufügen, dass D. Dahlstroms gründliche Studie des Wahrheitsbegriffs beim frühen Heidegger, dessen spätere 'Kehre diese Stimmung für viele verbürgen soll, letzterer die sachliche Berechtigung entzieht. Dahlstrom argumentiert, dass die transzendentalphilosophischen Probleme auch nach Heideggers Kehre zu einem Philosophieren in der Nähe von Dichtung und Glaube bestehen bleiben. Das hat für diesen Autoren zur Folge, dass die Auffassung von Wahrheit als vortheoretischer Erschlossenheit und Wahrheit als Aussagewahrheit letztlich als gleichursprünglich anerkannt werden müssen. Dahlstrom, Das logische Vorurteil, 20, sowie Kap. 5. 
Geschichte nicht über lange Jahrhunderte die Idee vorgeherrscht hätte, dass die Notwendigkeit von Glauben und Vertrauen durch so genannt sicheres Wissen überwunden werden kann und muss. Diese Vorstellung ist allerdings nicht länger selbstverständlich, wenn sie es denn jemals war: für viele Menschen heute scheint der Gedanke nur schwer nachvollziehbar, warum kognitive Vertrauenswürdigkeit allein wissenschaftlichen Weltzugängen und nicht z.B. auch mythischen oder religiösen zukommen sollte. Eine solche Skepsis ist philosophisch berechtigt. Denn offenkundig ist es wenig sinnvoll, die Vertrauenswürdigkeit eines so genannt wissenschaftlichen Weltbildes - im Gegensatz zu spezifischen wissenschaftlichen Theorien! wissenschaftlich erweisen zu wollen, wenn denn Vertrauen in die Fähigkeiten des menschlichen Verstandes notwendige Bedingung und Voraussetzung jeder theoretisch-begrifflichen Aktivität ist. Dazu kommt, dass ein generalisiertes Wissenschaftsvertrauen nur allzu leicht fehlgeleitet werden kann, wie, falls denn nötig, ein Blick auf die zivilisatorischen Katastrophen des vergangenen Jahrhunderts in Erinnerung bringen kann. Man braucht die grundsätzliche Berechtigung wissenschaftlicher Zugänge und Theorien in keiner Weise in Frage zu stellen um zu sehen, dass ein szientistischer Exklusivitätsanspruch auf kognitive Vertrauenswürdigkeit in Sachen Welterschliessung und Lebensgestaltung alles andere als selbst-evident ist und potentiell unmenschliche Folgen hat.

Auf diesem Hintergrund stellt sich somit die zentrale Frage nach einem Verständnis von Vertrauen, von dem her Wesen und Grenzen distinkter gesellschaftlicher Leistungssphären und der von ihnen geprägten Vertrauensformen und institutionellen Vertrauensvollzügen sichtbar gemacht werden können. Wenn es gelingt, Vertrauen als allgemein-universaler Modus philosophisch-ontologisch zu entfalten, haben wir zugleich eine transzendental-kritische und hermeneutisch-anthropologische Grundkategorie gewonnen, die allen unterscheidbaren, empirischen und historischen Vertrauensformen als deren Ermöglichungsbedingung zugrunde liegt. ,Vertrauen stünde in dieser Hinsicht auch nicht völlig alleine da. Ähnlich verhielte es sich mit anderen >Phänomenen oder historischen Phänomene unter anderen sind. Wie B. Cooper mit Bezug auf den in dieser Hinsicht äusserst fruchtbaren Denkansatz Voegelins summarisch festhält: »[O]ne does not confront history, politics, society, etc., as one would confront a thing. One participates in the object of study; one is a part of a larger whole. ${ }^{16}$

\footnotetext{
${ }^{16}$ Barry Cooper, The Political Theory of Eric Voegelin, Lewiston 1986, 2.
} 


\section{Ein Spektrum von modalen Aspekten}

Das eben angeführte Zitat artikuliert nun eine zu entfaltende Vermutung, die in der bisherigen Diskussion bloss am Rand zur Sprache gekommen ist. Die Vermutung ist: Vertrauen ist nicht der einzige modale Aspekt gelingenden Lebens. Neben Vertrauen sind andere Bedingungen der Möglichkeit von Erfahrung zu benennen, die diesem Aspekt sachlich svorausgehen genannt. Das Vertrauensthema würde sich nicht stellen, wenn es keinen `Raum Voraussetzungen menschlichen Daseins gäbe. Tote Menschen und untergegangene Gesellschaften haben keine Vertrauensprobleme, und noch weniger, wenn sie sich nirgendwo aufhalten. Aber dasselbe lässt sich auch sagen mit Bezug auf `Geschichte, Politik, Gesellschaft, etc. . Auch hier treffen wir auf Substantive, deren Verdinglichung theoretisch-begriffliche Probleme schafft. Dennoch sind diese Begriffe nicht einfach logisch-analytische `Fiktionen`, die für das Denken zwar unverzichtbar sein mögen, aber mit der `Realität $<$ nichts zu tun haben. Denn historisch-kulturelle Bedingungen konstituieren unsere Welt und ermöglichen den menschlichen Lebensvollzug nicht weniger als räumlich-geometrische und biotische Bedingungen. Der theoretisch artikulierbare Sinn unseres Daseins erschöpft sich nicht in snatürlichen Kategorien. Anders gesagt - im Idiom der klassisch-griechischen Philosophie gesprochen - partizipiert jedes Ding, einschliesslich das `Ding`Mensch, an einer umfassenden Seinsordnung. Diese Idee hat die menschliche Imagination und das Denken seit jeher in irgendeiner Form beschäftigt. Obwohl die Beschäftigung mit dem Sein und seiner Ordnung ihren frühesten dokumentierten Anfängen auch immer schon in Verruf stand, ‘blosse Spekulation $<$ zu sein, steht dahinter die kaum abzuweisende Intuition, dass unser Dasein verschiedene Dimensionen oder >Vollzugsebenen hat. Zur Illustration: Reduziert auf seine srein biotische oder organische Dimension, kann das menschliche Leben noch nicht einmal so genannt werden. ${ }^{17}$ Das Ganze der menschlichen Erfahrung und Existenz mag sich zwar dem theoretischen Zugriff entziehen, aber es lässt sich auch nicht theoretisch reduzieren. Voegelin bringt die Sache daher präzise auf den Punkt, wenn er schreibt: »Participation in being ... is not a partial involvement of man; he is engaged with the whole of his existence, for participation is existence itself. ${ }^{18}$

\footnotetext{
${ }_{17}$ Zur klassischen Unterscheidung von zoë und bios cf. Giorgio Agamben, Homo Sacer. Il potere sovrano e la nuda vita, Torino 1995.

${ }^{18}$ Eric Voegelin, Israel and Revelation, hg. v. Maurice P. Hogan, Columbia 2001, 39. Die deutsche Übersetzung trifft den Sinn der Passage nur ungefähr: "Die Partizipa-
} 
Nun haftet unter heutigen, so genannt postmetaphysischen Denkvoraussetzungen derVorstellung einer grundlegenden Seinsordnung zweifelsohne der Beigeschmack des Altertümlichen oder des noch Schlimmeren an. Wenn auch in vielfach gebrochener Form, wirft die Epoche und Idee der Aufklärung noch immer ihr Licht/Schatten auf diesen gesamten Problemkreis. Die Vorstellung einer ’kosmischen< Seinsordnung will sich scheinbar ganz und gar nicht mit der dynamischen Weltsicht einer westlich-modernen Gesellschaft, und ebenso wenig mit einem liberal-demokratischen Ethos der individuellen Selbstbestimmung, vertragen. Insbesondere die unentrinnbare Geschichtlichkeit und kulturelle Vielgestaltigkeit menschlichen Lebens scheint dem vormodernen Erfahrungshorizont traditionaler Gesellschaften - symbolisiert durch einen statischen Kosmos - diametral entgegengesetzt zu sein. Doch führt dieser Eindruck bei näherem Hinsehen zu mehr Fragen und Problemen, als einer davon genährten Sicht der Dinge lieb sein könnte. Warum? Die Beantwortung dieser Frage ist zugleich eine mögliche Antwort auf die Frage, ob der hier verfolgte ontologisch-modale Denkansatz nicht selber metaphysischer Natur und daher schlicht obsolet ist.

Die welterschütternde 'Entdeckung des achtzehnten Jahrhunderts einer kulturell und historisch irreduziblenVielfalt menschlicher Lebensformen ist im Gegensatz zum kulturell-historischen Aspekt aller Erfahrungswirklichkeit zweifellos selbst ein kulturhistorisches Ereignis oder zeitgeschichtliches Phänomen. Die Geschichtlichkeit aller Erfahrung ist dagegen eine allgemein-universale Bedingung, die, im Zusammenspiel mit anderen modalen Bedingungen, diese Entdeckung erst möglich macht. Anders als eine bestimmte theoretisch-begriffliche Analyse dieses unentrinnbaren Aspekts, ist die Geschichtlichkeit der Erfahrung selber nicht historisch kontingent oder variabel. Selbst noch oder gerade ein konsequenter Kulturrelativismus setzt die Geltung der These voraus, dass jeder menschliche Lebensvollzug zu allen Zeiten einen kulturhistorischen Charakter aufweist, wodurch der Eindruck einer unüberschaubaren Vielfalt von Lebensformen seine nachhaltige Wirkung überhaupt erst entfalten kann. Die historische Variabilität alles Erfahrbaren (Was) und die ontische Konstanz der geschichtlichen Dimension von Erfahrung (Wie) bedingen sich also gegenseitig. Wenn sich der strukturelle Sachverhalt der Geschichtlichkeit aller menschlicher

tion am Sein jedoch ist nicht eine Episode im Leben des Menschen, sondern nimmt seine gesamte Existenz in Anspruch, denn seine Partizipation ist seine Existenz selbst.", ders., Die kosmologischen Reiche des alten Orients - Mesopotamien und Ägypten, München 2002, 39. 
Erfahrung dem begrifflichen Denken einer Gesellschaft oder Epoche erst einmal aufgedrängt hat, prägt er notgedrungen auch die wissenschaftliche Rekonstruktion einer historischen Vergangenheit, in der dieses Bewusstsein so nicht existierte. Wir können deshalb nicht nur von der Vorgängigkeit von 'Vertrauen`, sondern ebenso von der Vorgängigkeit von `Geschichter sprechen. Dass das faktische Bewusstsein dieses modalen Sachverhalts subjektiv, d.i. von Mensch zu Mensch und Kultur zu Kultur sehr unterschiedlich ausfallen kann, ändert wohlverstanden nichts an der Tatsache. ${ }^{19}$

Vertrauen ist also nicht die einzige Ermöglichungsbedingung menschlichen Lebens. Wenn von gelingendem oder beschädigtem Leben die Rede ist, wird einerseits vorausgesetzt, dass die Bedingungen organischen Lebens deskriptiv vorhanden sind, und zum anderen, dass dieses Leben in weiter zu differenzierenden normativen Hinsichten (z.B. kulturell-historisch, ökonomisch, ästhetisch, rechtlich, moralisch, religiös etc.) gegenteilige Qualitäten annehmen kann. ${ }^{20}$ Es lassen sich entsprechend pränormative und normative Ermöglichungsbedingungen des menschlichen Lebens unterscheiden, die im Aspekt des Vertrauens vorausgesetzt sind. Kants summarische Umschreibung der fundamentalen Unterscheidung, die hier im Blick ist, lautete bekanntlich: „Der bestirnte Himmel über mir, und das moralische Gesetz in mir. ${ }^{21}$ Menschliches Vertrauen ist mit anderen Worten unweigerlich an pränormative (`natürliche`) Existenzbedingungen wie an normative ('logische und `kulturelle`) Vollzugsbedingungen gebunden.

In der hier entfalteten Perspektive lassen sich Natur und Freiheit, Realität und Idealität, Faktum und Norm, Kausalität und Sinn/In-

\footnotetext{
19 Auch Voegelin verfällt manchmal der shistoristischen Tendenz, Konstanz und Dynamik als konträre Gegenteile zu bestimmen. Vgl. die Bemerkung von P.J. Opitz, dergemäss sich Realität für Voegelin als nicht konstant erweist, "sondern jenen Prozesscharakter hat, den wir `Geschichte` nennen.» Peter J. Opitz/Gregor Sebba, The Philosophy of Order. Essays on History, Consciousness and Politics, Stuttgart 1981, 61. Doch diese Bestimmungen schliessen sich eben nur dann aus, wenn sie (prädikativ) auf ein und dasselbe Was bezogen werden. In modaler Hinsicht bedingen sich Konstanz und Dynamik aber gegenseitig, da der Geschichtscharakter von Realität im Gegensatz zum jeweiligen subjektiven Bewusstsein davon - selber konstant ist. Dooyeweerd fragt somit zu Recht: "How could we speak of historical development if its historical character itself were a variable phenomenon, dependent on a particular type of civilization in a particular phase of its development? « Dooyeweerd, A New Critique of Theoretical Thought, 2, 221.

${ }^{20}$ Wir sagen zum Beispiel, dass Leben und Denken bestimmter Kreise rrückwärts oder 'vorwärtsgerichtet ist, worin immer schon ein normatives Moment steckt.

${ }^{21}$ Immanuel Kant, Kritik der praktischen Vernunft. Grundlegung zur Metaphysik der Sitten, hg. v. Wilhelm Weischedel, Zürich 1977, A:289, 300.
} 
tentionalität, etc. nun aber nicht von einander trennen oder gegeneinander abschliessen (dies gegen Kant, der das Sein und das Sollen auf univoke (Weise voneinander geschieden haben wollte). ${ }^{22}$ Wenn zum Beispiel von einem exzessiven Vertrauen die Rede ist, spielt unweigerlich ein ‘ökonomischer Aspekte hinein, in einem solidarischen Vertrauen hingegen eine >moralische< Dimension. Vertrauen ist mit all diesen normativen Aspekten ranalogisch verbunden und überhaupt nicht ohne sie greifbar. ${ }^{23}$

Entscheidend für die Plausibilität einer Theorie modaler Aspekte ist nun wie gesagt, dass letztere nicht mit Dingen, Ereignissen, Handlungen, Prozessen, gesellschaftlichen Institutionen, Ideen, etc., also mit dem Was realer oder idealer Entitäten, verwechselt werden. Dieser Verwechslung zu entgehen fällt zugegebenermassen nicht immer leicht. Gerade wenn von einer modal gegliederten 'Seinsordnung die Rede ist, kann sich die Vorstellung einer kosmischen Hierarchie von Wesenheiten leicht aufdrängen. Eintagsfliegen leben einen Tag, Menschen etwas länger, Zivilisation bestehen noch länger, und der Kosmos überdauert alles - bis er vielleicht selbst zu Asche wird und ein neuer Zyklus von Werden und Vergehen beginnt. Die Seinsmacht dieser Dinge bezüglich ihrer Stabilität und `Lebensdauer ist also äusserst unterschiedlich. Die Vorstellung liegt nahe, dass alles, was innerhalb des menschlichen Horizonts begegnet - vom Gras bis zu den Gestirnen und den sie regierenden Göttern, vom Ephemeren bis zum Unvergänglichen - auf einer Skala der Dauer existiert, die zugleich eine Rangordnung von realem und idealem Sein, bzw. eine ontologische und eine axiologische chain of being suggeriert. Was weiter soben in der Seinsskala ist, muss eo ipso verlässlicher und realer sein. Die Teilhabe des Tiefergestellten an der Seinsmacht des Höhergestellten beinhaltet dann immer auch die Angst vor dem Absinken ins Nichts und die versuchte Absicherung gegen dieses Absinken. Cooper fasst diese ’kosmische Primärerfahrung (Voegelin) mit folgenden Worten zusammen: »Men die; women and fields may be barren; cities fall to armies and earthquakes; eclipses occur and equinoxes precess. Accordingly, rituals have been enacted on a periodic basis to retune the society with the cosmic rhythms...«. ${ }^{24}$

\footnotetext{
${ }^{22}$ Ein anschauliches Beispiel für die Kohärenz von Natur und Freiheit bietet der analogische Begriff der juridischen Kausalität, insofern hier auch noch die Unterlassung einer Handlung rechtliche Wirkung haben kann.

23 "The modal meaning of a law-sphere can only disclose itself in the intermodal coherence of meaning of all the aspects" Dooyeweerd, A New Critique of Theoretical Thought, 2, 150.

${ }^{24}$ Cooper, The Political Theory of Eric Voegelin, 168.
} 
Dennoch zeigt sich für Voegelin in der geschichtlichen Ausdifferenzierung dieses kosmologisch->kompakten` Bewusstseins, wenn auch nicht in allen religiösen und philosophischen Traditionen gleichermassen, dass alle Dinge, selbst die unsterblichen Götter, bestimmten Ermöglichungsbedingungen, und damit auch bestimmten Grenzen unterliegen. (Auf Voegelins Analyse der Rolle jüdischen und christlichen Denkens in der Herausbildung des Bewusstseins von sadikaler Transzendenz gehe ich weiter unten noch kurz ein.) Mit den modalen Aspekten der Erfahrung, wie sie von Dooyeweerd et al. herausgearbeitet wurden, sind nun eben die universalen >Gesetzer oder Bedingungen für das Auftreten, Gedeihen und Verschwinden aller Dinge unter der Sonne gemeint. ${ }^{25}$ Noch allgemeiner: Sie sind das, was die Identität und Dauer aller zeitlich begrenzten SSubjekter ermöglicht - und deshalb von qualitativ anderer Natur als diese. Mit anderen Worten: Die Ordnungsdimension der faktischen Dauer und die Ordnungsdimension der Geltung sind nicht ineinander überführbar.

Modale `Gesetze`, d.h. universale Bedingungen menschlicher Lebenswirklichkeit gehören der zweiten Ordnungsdimension an. Sie können determinierende oder normativ-orientierende Kraft haben im mythischen Denkhorizont des antiken Griechenlands einerseits symbolisiert durch die chtonischen (irdischen<) oder uranischen (’himmlischen`) Götter der Naturreligion, andererseits durch die olympischen Götter der Kulturreligion. ${ }^{26}$ Der biotische Aspekt des Lebens kann wiederum zur Illustration des Sachverhalts dienen: Natürliche Lebensprozesse sind unweigerlich durch metabolische Auf- und Abbauprozesse auf molekularer Ebene bestimmt und führen über kurz oder lang zum Tod der Kreatur. Und den physischen Gang der Gestirne können wir ebenso wenig bestimmen. Gesellschaftliche Ressourcen wie Treibstoffe und Gesetzesbestimmungen können dagegen, innerhalb bestimmter Grenzen, ökonomisch oder unökonomisch sein, oder gerecht oder ungerecht verteilt bzw. umgesetzt werden. Bestimmende `Gesetze haben also konstitutiven, normative Prinzipien hingegen konstitutiven und regulativen Charakter. Normen kann man - wiederum innerhalb gewisser Grenzen zuwiderhandeln, d.h. ohne die Konsequenzen davon unmittelbar selber zu spüren zu bekommen. Die Regierung eines Staates kann

${ }^{25}$ M. Jandl spricht von `Funktionsdimensionen in: Martin J. Jandl, Praxeologische Funktionalontologie. Eine Theorie des Wissens als Synthese von H. Dooyeweerd und R.B. Brandom, Frankfurt a.M. 2010.

${ }^{26}$ Herman Dooyeweerd, Reformation and Scholasticism in Philosophy. The Greek Prelude, Lewiston 2004, 4. 
sich mit Gewalt gegen die eigene Bevölkerung wenden, aber nicht ohne dass der betroffene Staat über kurz oder lang auseinanderbricht.

Modale Aspekte der Erfahrung, wenn es sich denn wirklich um solche handelt, ${ }^{27}$ sind wechselseitig irreduzibel. Der hier umrissene, nicht-reduktive Denkansatz steht und fällt mit dieser Grundannahme. Daraus folgt, dass Vertrauensprozesse nicht auf biotische Lebensprozesse reduzierbar (oder umgekehrt) sind, Lebensprozesse nicht auf physische Energieprozesse (oder umgekehrt), >moralischer Beziehungen nicht auf rechtliche, und beide zusammen nicht auf ökonomische Interaktionen. Die Irreduzibilität dieser modalen Aspekte kommt auf theoretischer Ebene dadurch zum Ausdruck, dass jeder einen strukturellen Sinnkern hat, der letztlich undefinierbar ist. So ist zum Beispiel der Sinnkern des Raumaspekts die der ’kontinuierlichen Ausdehnung; der nicht weiter zerlegbare Kern des Rechtsaspekts besteht hingegen darin, dass $\mathrm{x}$ zukommt, was $\mathrm{x}$ zusteht. ${ }^{28}$ Was aber ist nun der Sinnkern des Vertrauensaspekts?

\section{Der modale Aspekt des Vertrauens}

Der undefinierbare Kern des Vertrauensaspekts, den Dooyeweerd für heutige Ohren vielleicht irreführend als Glaubensaspekt bezeichnete, kann wohl am besten mit fiducia, confidence, faith, etc. ausgedrückt werden..$^{29}$ Auch hier, ebenso wie beim Ausdruck >Leben $<$ oder > Geschichter, kann sich leicht eine Verwechslung von faktischem Was und modalem Wie aufdrängen. In Übereinstimmung mit dem bisher Gesagten beziehen sich diese Bezeichnungen des fiduziären Aspekts aber nicht in erster Linie auf einen Bewusstseinsakt bzw. auf eine mehr oder weniger kognitiv-epistemisch gesicherte Glaubenseinstellung zur Welt. Der modale Sinnkern von Vertrauen steht somit

\footnotetext{
${ }^{27}$ Dooyeweerd unterscheidet und analysiert deren fünfzehn, es könnten prinzipiell aber auch mehr oder weniger sein.

${ }^{28}$ Auch P. Tillich hat diese sprimitive Bedeutung im Auge, wenn er vom stributiven Charakter von Recht und Gerechtigkeit spricht. Cf. Paul Tillich, Liebe, Macht, Gerechtigkeit, Tübingen 1955, 63-64. >Tribution` wäre dem von Dooyeweerd nur ungern verwendeten Ausdruck /Retribution denn auch vorzuziehen - er vermeidet die einseitig strafrechtlichen Konnotationen - wenn es sich dabei nicht um einen Neologismus handelte.

${ }^{29}$ Cf. Herman Dooyeweerd, Die Philosophie der Gesetzesidee und ihre Bedeutung für die Rechts- und Sozialphilosophie, Archiv für Rechts- und Sozialphilosophie 53, 3; 4 (1967), 1-30; 465-513, 4. An dieser Stelle ist auch von der "pistischen « Dimension der Erfahrung die Rede.Vielleicht hat Dooyeweerd hier vom Hebräischen her gedacht - in dem bekanntlich nicht scharf zwischen Glauben und Vertrauen geschieden wird - aber Griechisch formuliert.
} 
auch nicht in Kontrast zu >Meinen oder >Hoffen Seite, und /Wissen auf der anderen Seite. Diese Auffassung würde das Wie eines irreduziblen Aspekts des menschlichen Erfahrungshorizonts und Lebensvollzugs namens Vertrauen bloss wieder mit dem Was eines logisch-begrifflich objektivierten Sachverhaltes, d.h. mit einer subjektiven mentalen Einstellung, deren Korrelat ein objektiv-propositionaler Gehalt ist, verwechseln. Dann gerät aber aus den Augen, dass Glauben, Meinen, Hoffen und Wissen Vertrauen in die Erkennbarkeit unserer Welt immer schon implizit voraussetzt.

Auch ist mit den erwähnten Ausdrücken fiducia, faith, etc. nicht notwendig eine positive Zukunftserwartung verbunden. Vielmehr verweisen sie die philosophische Analyse von Vertrauen auf das unhintergehbare Moment der (Un)gewissheit in jedem möglichen Lebensvollzug und Weltzugang.Vertrauen als universaler Modus der Erfahrung und des Lebensvollzugs ist vorausgesetzt in allem Tun und Lassen - etwa als Vernunftglaube, wo Welt intelligibek gemacht oder wissenschaftlich erschlossen werden soll, oder als Gemeinschaftsvertrauen, wo zwischenmenschliche und institutionelle Beziehungen existieren sollen, oder als Selbstvertrauen, wo ein `Ich statt ein `Es< sein soll. Doch versteht es sich von selbst, dass jeder (mögliche) Ausgangspunkt einer Erkenntnis oder eines rationalen Arguments, wie überhaupt eines jeden individuellen oder gemeinschaftlichen Lebensvollzugs, aus verschiedenen Ursachen und Gründen erschüttert und unsicher werden kann. Wie Descartes cogito oder `Ich denke‘, das ein unlimitiertes Vertrauen in die Gewissheit des logisch-rational Unbezweifelbaren zum Ausdruck bringt, kann auch (das Vertrauen in) jede Gemeinschaft und soziale Ordnung zerbrechen.

Daraus folgt: Die universale Strukturnorm des fiduziären Aspekts hat die adäquate Unterscheidung zwischen Gewissem und Ungewissem, zwischen Verlässlichem und Unverlässlichem, Vertrauenswürdigem und Nicht-vertrauenswürdigen im Blick. Alles was uns von innen oder aussen entgegenkommt, und alles was wir selbst hervorbringen, untersteht dieser Norm. Demzufolge ist das einzige, was im Leben gewiss ist, dass wir - besser oder schlechter - zwischen Gewissem und Ungewissem unterscheiden müssen. ${ }^{30}$

\footnotetext{
${ }^{30}$ Damit ist auch impliziert, dass menschliches Leben nicht per se als sungesichert bezeichnet werden kann. >Unsicherheit wird erst zum Problem, wo, aus welchen Ursachen und Gründen auch immer, nicht adäquat zwischen Gewissem und Ungewissem, bzw. zwischen Vertrauenswürdigem und Nichtvertrauenswürdigem unterschieden werden kann. Dieses Nicht-unterscheiden-können kann je nach Situation tiefer oder weniger tief gehen. Aber dass menschliches Leben nicht instinkt-gesichert ist, stellt so betrachtet noch keinen Mangel oder Defizit dar, sondern zeichnet gerade Leben als menschliches Leben aus. Umgekehrt ist hier aber auch ausgeschlossen, dass
} 
Das gilt auch für das rabsolut <Vertrauenswürdige in den Religionen. Vertrauen gibt es nicht ohne angemessenes oder unangemessenes Vertrauen. Alles menschliche Leben spielt sich zwischen diesen beiden normativen Polen ab. Deren Unterscheidung nehmen wir aber unweigerlich unter distinkten Gesichtspunkten oder Aspekten vor, etwa zwischen dem was sensorisch-psychisch, logisch-analytisch, historisch-kulturell, sozial, ökonomisch, ästhetisch, rechtlich, moralisch und religiös gewiss (vertrauenswürdig) oder ungewiss (nicht vertrauenswürdig) ist. Diese Vertrauenshinsichten sind dabei nicht nur auf /Welt` gerichtet, sondern korrelieren immer auch mit einer entsprechenden Form von Selbstvertrauen. ${ }^{31}$

Damit tritt ein letzter wichtiger Punkt hervor, auf den in diesem Abschnitt noch etwas ausführlicher eingegangen werden soll. Weiter oben wurde gesagt, dass normative Aspekte der Erfahrung zugleich konstitutive und regulative Bedeutung für das gelingende menschliche Leben haben. Wir müssen zwischen Gewissem und Ungewissem unterscheiden (fiduziärer Aspekt), zwischen Recht und Unrecht (juridischer Aspekt), zwischen Liebe bzw. solidarischer Zuwendung und aggressiver Indifferenz oder Zurückweisung (moralischer Aspekt), zwischen Exzess und dem Vermeiden von Exzess (ökonomischer Aspekt), zwischen Widersprüchlichem und Nichtwidersprüchlichem (logisch-analytischer Aspekt), etc. Gelingendes Leben kann nur vollzogen werden, wenn die Individuen und Gemeinschaften einer Gesellschaft in allen diesen Hinsichten (zumindest manchmal) die richtigen Unterscheidungen treffen. Diese Hinsichten treten nun wohlgemerkt nicht 'nebeneinander auf, sondern sind analogisch miteinander verbunden: Rechtsvertrauen oder -gewissheit steht Rechtsungewissheit gegenüber, Liebesvertrauen oder-gewissheit steht im Kontrast zu Liebesungewissheit, logische Gewissheit im Gegensatz zu logischer Ungewissheit, etc.

Aber nun lässt sich diese Reihe normativer Gegensätze im Aspekt des Vertrauens fortsetzen in den Bereich der pränormativen ('natürlichen`) Aspekte der Erfahrungswirklichkeit. Wir sprechen von

\footnotetext{
ein subjektives Streben nach Allbeherschung der Um- und Mitwelt die strukturelle Notwendigkeit der Unterscheidung zwischen Gewiss und Ungewiss aufheben könnte.

31 Wenn ich recht sehe, entspricht in der breitangelegten Studie von Godfrey das Vertrauen in unser subjektives Vermögen, in allen genannten Hinsichten das Vertrauenswürdige vom Nichtvertrauenswürdigen prinzipiell (de facto freilich nicht immer zuverlässig) unterscheiden zu können, dem was er selber "openness-trust «nennt; z.B. Godfrey, Trust, 293. Je mehr ein solches Vertrauen fehlt oder dessen Möglichkeit bestritten wird, desto mehr verschiebt sich das Bild in Richtung eines "nihilism of understanding and motivation and a skepticism of thought." (295)
} 
lebenden und von toten Elementen einer Denktradition oder Stilrichtung (biotische Analogie), um von der geistigen Kohäsion einer Gesellschaft (physische Analogie), etc. Sinn und Bedeutung dieser prima facie empirisch-deskriptiven Ausdrücke sind hier zugleich normativer Art, da sie sich nicht primär auf biotische oder physische, sondern auf soziokulturelle Aspekte eines in sich komplexen, multimodalen Sachverhaltes beziehen. An toten Traditionselementen und veralteten Lebensformen kann man sich nicht verlässlich orientieren. Aber wann ist eine Tradition und das Vertrauen in sie tot? Wissenschaftliche Theorien, historische Rechtstraditionen und religiöse Dogmen und Institutionen vergehen nicht von selbst, und soziale Anomie ist nicht dasselbe wie physische Entropie. Solche Dinge stehen in unverbrüchlichem Zusammenhang mit kulturellen und logisch-theoretischen Handlungsnormen. Man kann sie deshalb als Artefakte bezeichnen. Dennoch lassen sie sich nicht einfach per Dekret oder Willensentscheidung in die gewünschte Form bringen. Lebendiges Vertrauen in Begriffe, Gemeinschaftsformen und Institutionen lässt sich nicht planmässig herstellen ‘. Alle diese Dinge haben in modaler Hinsicht immer auch eine sorganischer Dimension, ein 'Eigenleben<, das nicht im planenden Herstellen und Handeln führender `kultureller Organe aufgeht. Denn schliesslich sind bisher noch alle menschlichen Zivilisationen untergegangen. Biotische Analogien in der modalen Struktur von Vertrauen und allen anderen normativen Aspekten der Erfahrung haben somit ihren kulturellen und spirituellen Sinn. Dennoch, oder gerade deshalb, müssen sie von snatürlichen (biotisch qualifizierten) Prozessen qualitativ unterschieden werden.

Normativ verlässliche Orientierung an natürlichen Analogien im Bereich von Kultur und Gesellschaft erscheinen so gesehen als kaum vermeidbar. ${ }^{32}$ Dagegen könnte nun vielleicht eingewendet werden, dass die aufgeführten Analogien (ıtote Theorien oder Traditionselemente) auf blossen Redeweisen beruhen, auf die man ebenso gut verzichten könnte. Dagegen spricht aber schon die Tatsache, dass es sich bei diesen Ausdrücken ihrerseits um tote (!) Metaphern handelt. Modale Analogien lassen sich nicht so leicht umgehen; selbst noch der Ausdruck der `Metapher (Gr. meta-phéro, hinübertragen) basiert schliesslich auf einer räumlichen Analogie in der sprachlichen Dimension menschlicher Erfahrung. Man müsste deshalb schon auf Sprache überhaupt verzichten können, um solchen Analogien und

${ }^{32}$ Weitere Beispiele dafür sind schnell bei der Hand, so etwa der Begriff einer smechanischen` Kausalität, der ohne die Analogie zur Maschine als kulturellem Artefakt nicht zu denken ist. 
der Frage nach ihrer Verlässlichkeit in Leben und Denken auszuweichen. Kurzum: die modale Struktur von Vertrauen scheint die Struktur der pränormativen Aspekte notwendig vorauszusetzen und in sich zu renthalten . Normativ verlässliche Orientierung in unserer Welt basiert auf der vortheoretischen Intuition snatürlicher Analogien, die sich nicht - trotz des Ideals einer tiefeingewurzelten westlichen Denktradition - in ein reines Sein und ein reines Sollen auseinanderziehen lassen.

Der psychische Aspekt des Fühlens und Empfindens, die affektive Dimension der Erfahrungswirklichkeit, spielt hier zweifellos eine entscheidende Rolle. In unserem Diskussionszusammenhang zeigt sich die Bedeutung dieses Aspekts besonders in der wiederkehrenden Frage, ob Vertrauen eher ein Urteilen oder ein Fühlen ist. Auf dem Hintergrund des vorliegenden Denkansatzes existiert nun jedoch nicht die geringste Notwendigkeit, sich entweder für das eine oder das andere entscheiden zu müssen. Denn der irreduzible Sinnkern von Vertrauen hat unverbrüchliche strukturelle Analogien sowohl im affektiven wie im logisch-analytischen Aspekt der Erfahrung. Man kann sich das folgendermassen klar machen: Ein konkreter Vertrauens- oder Glaubensakt muss weder gefühlt noch logisch-analytisch als solcher identifiziert werden um tatsächlich vorzuliegen. ${ }^{33}$ Aber wenn (erschüttertes) Vertrauen weder gefühlt noch logisch objektiviert werden könnte, wäre der vermeintlich irreduzible Sinnkern von Vertrauen einfach leer. Gefühltes Vertrauen bzw. empfundener Vertrauensverlust und Angst braucht nun wohlverstanden nicht auf einem expliziten oder auch nur impliziten Urteil der (mangelnden) Vertrauenswürdigkeit von x zu beruhen. Dennoch hat es durchaus intentionalen Charakter. Das heisst, es ist immer auf etwas gerichtet und somit nichts rein< Natürliches, das allein mit kausal-psycholo-

\footnotetext{
${ }_{33}$ Der Atomphysiker Werner Heisenberg, Der Teil und das Ganze. Gespräche im Umkreis der Atomphysik, München 1988,113, verweist auf eine Geschichte, die sein Kollege Nils Bohr zu erzählen pflegte, und die diesen Punkt anschaulich illustriert. Die Anekdote handelt von einem Mann, der ein Hufeisen über der Eingangstür seines Hauses angebracht hatte. Als ein Bekannter ihn fragte, ob er denn so abergläubisch sei zu glauben, dass ihm das Hufeisen Glück bringe, antwortete er: "Natürlich nicht; aber man sagt doch, dass es auch dann hilft, wenn man nicht daran glaubt."Was ist hier schiefgelaufen? Die logisch-analytische Selbstdistanzierung vom psychischen Aspekt des Vertrauens bzw. Glaubens verweist auf ein logisch qualifiziertes ,Wissen des Mannes, das eben diesen Aspekt zwar voraussetzt, ihn aber zugleich subjektiv unsichtbar macht. Der performative Selbstwiderspruch, der aus dieser geistigen Haltung resultiert, könnte als Folge eines logizistischen Hypervertrauens bezeichnet werden, das sich auf alles mögliche, auf wissenschaftliche Theorien nicht weniger als auf die Wirkung von Glücksbringern, erstrecken kann.
} 
gischen Begriffen geklärt werden könnte. ${ }^{34}$ Die logisch-analytische Identifizierbarkeit bzw. Unterscheidbarkeit dieses Etwas als etwas ist vielmehr die Voraussetzung für die Frage nach dessen Vertrauenswürdigkeit, d.h. der sachlichen Adäquatheit und normativen Rechtfertigung unseres Vertrauens in x. Diese Frage muss und kann sich nicht immer und überall gleich stellen; das >Gesetz der Liebe verlangt etwas anderes von uns als das `Gesetz` des ökonomischen Vorteils oder das `Gesetz^ der politischen Gerechtigkeit.

Freilich, wie wir uns lebenspraktisch an allgemein-modalen 'Gesetzen und normativen Prinzipien orientieren, hängt von den vorfindlichen Umständen und den typisch-relevanten, situationsbestimmenden Normen ab. Modale Normen entfalten ihre orientierende Kraft nicht in abstracto, sondern immer in situationsspezifischer und kulturell-historisch positivierter Form. Und hier beginnen offensichtlich auch die Schwierigkeiten. Denn allgemein-modale 'Gesetze und Normen werden von Menschen nicht automatisch richtig erfasst und positiviert. Das kann sehr verschiedene Ursachen und Gründe haben. Oft wird z.B. deren strukturelle Sinngehalt aus ideologischen oder religiösen Gründen verzerrt oder gänzlich negiert. So hat zum Beispiel das modale Gesetz des Werdens und Vergehens - >Alles was entsteht, vergeht $\_$-, dem jede physische Struktur als solche unterliegt, nicht nur rein konstitutiven oder fundierenden Charakter. Je nach Epoche und vorherrschendem religiösen Bewusstsein kann dieses Gesetz zur Leitnorm einer bestimmten Lebensorientierung oder Weltanschauung werden, die dem Gesellschaftsprozess eine eigene Richtung verleiht. Dass alle Dinge im physischen und biotischen Universum insofern gleich sind, dass das `Höchster wie das `Niedrigster dem Gesetz von Werden und Vergehen unterliegt, kann einerseits zum spirituell wirkmächtigen snatürlichen Symbol für kreatürliche Gleichheit und Mitgefühl werden. ${ }^{35}$ Wird der strukturelle Sinnkern des physischen Aspekts von Energie/Materie jedoch von allen anderen Aspekten der Erfahrung getrennt und damit wörtlich verabsolutiert - so dass das einzige, was Bestand hat, die Gewissheit ist, dass letztlich nichts Bestand hat und alles sim Fluss i ist - steht die permanente Revolution, der Klassenkampf, die >Anarchie der Meinungen` (W. Dilthey), oder

${ }^{34}$ Mit Dooyeweerd et al. könnte man deshalb sagen, dass ein konkreterVertrauensakt fiduziär qualifiziert, aber psychisch-affektiv fundiert ist. Vertrauen ১beginnt` im Gefühlsaspekt, aber endet nicht dort.

${ }^{35}$ Vgl. die Zeilen aus Robert Burns Gedicht > To a Mouse, on Turning Her Up in Her Nest with the Plough (1785): "I'm truly sorry man's dominion / Has broken Nature's social union, / And justifies that ill opinion / Which makes thee startle / At me, thy poor, earth born companion / And fellow mortal!« 
schlicht ein existentieller Selbstwiderspruch vor der Tür. Da nun schon allein die Vorstellung solcher Dinge schwer auszuhalten ist, droht zugleich jedoch die umgekehrte Entwicklung, die Voegelin als Re-Divinisierung des Kosmos und der Gesellschaft bezeichnet hatte. Damit gemeint ist eine Entwicklung des Denkens, die auf die ,Entgöttlichung< des antiken Kosmos und des imperialen Herrschers durch ein jüdisch-christliches Gottes- und Weltverständnis so reagiert, dass sie dem Menschen eine neue Identität im >rein ‘ diesseitig verstandenen Wirklichkeits- oder Gesellschaftsganzen verspricht. ${ }^{36}$ Das kann auf verschiedene Weise geschehen. Oft wurden biotischorganische Analogien zur weltanschaulich-ideologischen Plausibilisierung eines solchen Wirklichkeits- und Gesellschaftsvertrauens herangezogen.$^{37}$ Die Vollendung des Menschen (eines bestimmten Typs) in und durch >die Gesellschaft oder >den Staat anstatt durch die göttliche Gnade im Tod wurde nun nicht nur in immanenttotalitären Ganzheitsideologien verkündet, sondern entsprach gemäss Voegelin einem evolutionistischen Grundzug des neunzehnten Jahrhunderts überhaupt. Der Sinn einer philosophischen Analyse modaler Strukturen der Erfahrung besteht unter anderem darin, solche Entwicklungen begrifflich präzis nachzeichnen zu können.

\section{Keine modalen Aspekten ohne konkrete Phänomene: `Gesetzı und `Subjekt`}

Bisher war ausführlich von modalen Aspekten der Erfahrungswirklichkeit und des menschlichen Lebensvollzugs im Ganzen die Rede. Diese machen aber nur die eine Seite der Medaille aus. Auf der anderen Seite sind die unerschöpflich variablen, konkreten Natur- und Kulturphänomene, die durch sie ermöglicht werden. Dooyeweerd denkt die beiden Seiten in strikter und unverbrüchli-

\footnotetext{
${ }^{36}$ Cf. Eric Voegelin, Die Neue Wissenschaft der Politik: Eine Einführung, München 1959, 176. Für Voegelin verstärkt das sschwache Band des christlichen Glaubens die Last einer `kosmischen Ungesichertheit des Daseins noch zusätzlich. Er schreibt: „Das Leben der zu Gott hin geöffneten Seele, das Warten, die Zeiten der Dürre und Mattigkeit, der Schuld und Betrübnis, der Zerknirschung und Reue, der Verlassenheit und der gläubigen Hoffnung, der stillen Regungen von Liebe und Gnade, zitternd an der Schwelle einer Gewissheit, die, wenn sie gewonnen, ein Verlust ist, - gerade die Schwerelosigkeit dieses Gewebes mag sich als zu schwere Belastung für Menschen erweisen, die auf handfesten Besitz aus sind." (ebd.)

${ }^{37}$ Die Grundlegung eines >organisch-ganzheitlichen Wissenschaftsverständnisses in der deutschen Romantik wird in Kristian Köchy, Ganzheit und Wissenschaft: Das historische Fallbeispiel der romantischen Wissenschaft, Würzburg 1997, detailliert nachgezeichnet.
} 
cher Korrelation. ${ }^{38}$ Seinem religiösen Denkhorizont als überzeugter Calvinist und professionellen Hintergrund als Rechtstheoretiker entsprechend, gibt es für ihn keine >Gesetzer ohne >Subjekter. sSubjekt ist dabei alles, was faktisch (möglich) ist und in seiner typischen Funktionsweise durch modale ,Gesetzer bzw. Normen bestimmt ist. Die Ordnung der faktischen Dauer und die Ordnung der modalen Geltung werden nicht unabhängig von einander, sondern als strikt korrelierende Dimensionen menschlichen Bewusstseins gedacht.

Nun unterstehen selbstverständlich nicht alle Dinge den modalen 'Gesetzen und Normen auf dieselbe Weise. Naturphänomene wie Feuer und Eis sind in spezifischer Weise durch physische Gesetze bestimmt. Sie sind zuerst physische sSubjekte bzw. durch entsprechende 'Subjektfunktionen ‘ bestimmt. Ihr inneres `Konstruktionsprinzip ist nicht primär quantitativ-arithmetischer oder räumlich-geometrischer Art, selbst wenn der physische Aspekt natürlicher Ereignisse den quantitativen und den räumlichen Aspekt von Zahl und Raum im Aufbau menschlicher Erfahrungswirklichkeit unweigerlich voraussetzt. Gerade aufgrund seiner typischen Subjektfunktionen im physisch-chemischen Aspekt kann Feuer nun durch shöherstufig qualifizierte Subjekte wie Menschen auch für historisch-kulturelle Zwecke erschlossen werden. Feuer ist in Wirklichkeit also keineswegs etwas bloss Physikalisches, ${ }^{39}$ sondern ebenso ein - freilich äusserst zwiespältiges - Kulturgeschenk der Götter. Dinge wie Feuerproben und Kühlboxen sind im Gegensatz zu natürlichen Phänomenen wie Frost und Gewitter in pränormativen Subjektfunktionen fundiert, die aber nach kulturellen Normen objektiviert wurden. ${ }^{40}$

Zwei Konsequenzen folgen unmittelbar. Das Auftreten von natürlichen wie gesellschaftlichen Dingen, Ordnungsstrukturen und Prozessen ist immer auch an typische - und nicht bloss an allgemeinmodale - Gesetze und Normen gebunden, die allein für diese Art von x gelten. Zudem gilt für Gesellschafts- und Kulturphänomene,

\footnotetext{
${ }^{38}$ Dadurch ausgeschlossen ist sowohl ein splatonischer Universalien- oder Ideenrealismus wie auch ein moderner Nominalismus, dem bloss konkrete Einzeldinge als 'wirklich gelten.

${ }^{39}$ Die Abstraktion physikalischer Strukturgesetze unserer Erfahrungswirklichkeit verdankt sich nicht primär der 'natürlich -kausalen Entwicklung des menschlichen Gehirns, obwohl Abstraktion als konkrete Tätigkeit und Vermögen eine solche Entwicklung voraussetzt, sondern vielmehr der historisch-kulturellen Erschliessung der Möglichkeiten theoretisch-begrifflich Denkens.

${ }^{40} \mathrm{H}$. Berman weist auf die Feuerprobe als Teil der germanischen Rechtspraxis des 'Gottesurteils` hin: "[...] wenn die Verbrennungen richtig verheilten, war man exkulpiert." Harold J. Berman, Recht und Revolution. Die Bildung der westlichen Rechtstradition, Frankfurt a.M. 1991, 97.
} 
wie eben nochmals hervorgehoben wurde, dass ihr Wesen nicht 'natürlich vorgegeben ist, sondern dass sie erst durch formative Gestaltung oder Positivierung der sie typisierenden normativen Strukturprinzipien auftreten und aufrechterhalten werden können.

Der moderne Rechtsstaat, beispielsweise, wird durch eine typische Konfiguration modaler Aspekte ermöglicht, unter denen der rechtliche Aspekt hervortritt und leitende Bedeutung annimmt. ${ }^{41}$ Doch dieser rechtliche Aspekt menschlichen Daseins erschöpft sich nicht in den faktischen Institutionen und Praktiken des rechtlich-politischen Gemeinwesens oder der 'Zentralgewalt . Eine solche spositivistischer Auffassung der Dinge würde darauf hinauslaufen, dass ein Staat seine historischen und rechtlichen Ermöglichungsbedingungen in sich selber tragen und somit gewissermassen causa sui sein könnte. Das ist aber nicht nur theoretisch widersinnig, sondern hat auch sonst unschöne, weil potentiell totalitäre Konsequenzen, da dann eben keine normativen Rechtsprinzipien «über dem vorfindlichen Staatswesen rexistieren könnten, aufgrund derer die rechtspolitische (Un) Ordnung einer Gesellschaft als ungerecht oder sonst wie normativ deformiert kritisiert und bekämpft werden könnte. Ein faktisches Gemeinwesen mit seinen bestehenden Regeln und Gesetzen (Was) kann nicht mit Recht und Gerechtigkeit in modalem Sinn (Wie) identifiziert werden, ohne dass die Legitimität und damit auch die Vertrauenswürdigkeit dieser Gemeinschaft gleichzeitig in Frage gestellt ist.

Die grundlegende Unterscheidung zwischen `Subjekt und $>\mathrm{Ge}-$ setz ist kein idiosynkratisches Merkmal unseres Denkansatzes. Sie findet sich auch im Herzen antiker, mittelalterlicher und moderner Naturrechtstheorien. Das ist nun nicht weiter erstaunlich, wenn, wie hier vorgeschlagen, jede faktisch bestehende politische Rechts(un) ordnung einem qualitativ anderen `Gesetzı untersteht, das die Bedingung der Legitimität und Vertrauenswürdigkeit dieser Ordnung umfasst. ${ }^{42}$ Gleichzeitig muss aus modaler Perspektive gegen das traditionelle und moderne Naturrechtsdenken aber eingewendet werden, dass der Rekurs auf vermeintliche Prinzipien einer shöheren Gerechtigkeit der Moral keine theoretische adäquate Grundlage für die Kritik an der politischen Rechts(un)ordnung einer ausdifferen-

\footnotetext{
${ }^{41}$ Genau gesprochen ist der moderne Rechtsstaat als historisch-kulturelles Artefakt gemäss Dooyeweerd im historischen Aspekt fundiert, aber durch den juridischen Aspekt qualifiziert.Vgl. dazu kritisch Jonathan Chaplin, Herman Dooyeweerd. Christian Philosopher of State and Civil Society, Notre Dame 2011, 167ff.

${ }^{42}$ Diese antipositivistische Implikation eines universalen Rechtsaspekts des sozialen Lebens wird ebenso von Berman, Recht und Revolution, 138, unterstrichen.
} 
zierten Gesellschaft bieten kann. ${ }^{43}$ >Moralische< Kritik und Reformen können die Vertrauenswürdigkeit einer Staatsordnung nicht verbessern. Denn in einer ausdifferenzierten Gesellschaftsordnung ist der Staat eben in erster Linie durch rechtlich-politische und nicht etwa durch >moralische Normen typisiert, wie dies etwa Familien-, Verwandtschafts-, Nachbarschafts-, Freundschaftsbeziehungen und Lebenspartnerschaften sind. Der moderne Rechtsstaat kann nicht von derselben Art sein wie eine zivilgesellschaftliche Fürsorgegemeinschaft oder eine Gemeinschaft von $>$ Ich und Dur. ${ }^{44}$ Moralische Kritik am politischen Gemeinwesen misst den Staat irrtümlich an der strukturellen Solidaritäts- oder Liebesnorm eines anderen, >moralischen< Gemeinschaftstyps. Oder sie muss unmittelbar auf eine transzendente, $\mathrm{Ur}-\mathrm{Norm}<$ rekurrieren, die den modalen Horizont menschlichen Daseins gleichsam übersteigt. Die erste Option kommt einer so wohlmeinenden wie irregeleiteten Moralisierung bzw. Idealisierung von Politik gleich - mit der umgekehrt eine Politisierung und Verrechtlichung von Ich-Du-Beziehungen einhergeht. ${ }^{45}$ Beides schadet dem Vertrauen, das wir in den Staat als rechtspolitisch qualifizierte Gemeinschaft haben können (sollten). Die zweite Option basiert dagegen auf einer konzeptuellen Unmöglichkeit. Denn jeder theoretische Bezug auf eine transzendente Ur-Norm ist immer schon eine implizite oder explizite Positivierung derselben im Horizont des einen oder anderen modalen Erfahrungsaspekts bzw. einer typischen Interaktionssituation.

\section{Die normative Strukturtypik von Vertrauen}

Was impliziert das bisher Gesagte nun für die unzählig vielfältigen, konkreten Vertrauensformen und -feldern in unserer Erfahrung? Wenn es zutrifft, dass jede Sphäre einer ausdifferenzierten Gesellschaft ihre eigenen strukturtypischen Interaktionsverhältnisse, Beziehungs-

\footnotetext{
${ }^{43}$ So auch Brunner, Gerechtigkeit, Zürich 1943, 150.

${ }^{44}$ Autoritätsverhältnisse in Kirche und Staat, etc., sind durch Super-bzw. Subordination der Interaktanten, Ich-Du-Beziehungen aber durch Koordination der Beziehungsglieder qualifiziert. Dieser wichtige Unterschied wird von Godfrey m.E. nun aber so stark ontologisiert, dass er die kulturhistorische Tatsache der Interdependenz der jeweiligen Sphären und ihren spezifischen Vertrauenstypen aus den Augen zu verlieren droht. Durch die Zerschlagung ,feudaler Strukturen eröffnet z.B. die Entstehung des modernen Nationalstaats auch weit mehr Möglichkeiten für Ich-Du-Beziehungen als frühere gesellschaftspolitische Arrangements.

45 So auch Godfrey: "The threat to the worth of I-thou trust comes, as it were, from outside, from an interest in having I-thou trust cover all the dimensions of trust." Godfrey, Trust, 159.
} 
und Gemeinschaftsformen ausbildet, dann sind letztere ihrerseits von entsprechenden Vertrauensformen und -normen getragen, die nicht aufeinander zurückgeführt werden können. >Proper trust< oder seigentliches Vertrauen k kann nicht immer und überall dasselbe bezeichnen, wenn verschiedene persönliche und gesellschaftliche Güter im Spiel sind. ${ }^{46}$ Die Frage > Was tun wir, wenn wir vertrauen? kann dann nicht von diesen spezifischen Kontexten abgelöst werden. Was alle diese typisch verschiedenen Vertrauenskontexte oder -verhältnisse miteinander gemeinsam haben, kann dann aber auch nicht an dem einem oder anderen dieser Kontexte festgemacht werden. Es ist theoretisch wie praktisch nur abwegig, einen bestimmten Beziehungstypus bzw. eine bestimmte Interaktionssituation - z.B. die zwischen Kleinkind und primärer Bezugsperson, die zwischen ökonomisch->rationalen` Subjekten, oder die zwischen `Fremden $\prec-$ als Paradigma für Vertrauen süberhaupt $\mathrm{zu}$ nehmen. Verschiedene Arten von sozialen Vertrauensbeziehungen unterstehen ihren eigenen strukturtypischen Normen, die nicht unter eine allgemeine rational-ökonomische oder rational-moralische Metanorm zu bringen sind, ohne dass die realen Unterschiede zwischen konkreten Beziehungs- und Vertrauenstypen begrifflich nivelliert werden - und damit dem menschlichen Lebenshorizont theoretische Gewalt angetan wird. Was im einen Kontext normativ adäquat oder srationak ist, muss es im anderen nicht sein. Semiotisch qualifiziertes Vertrauen in die Wegbeschreibung eines Fremden ${ }^{47}$ ist nicht dasselbe wie politisch qualifiziertes Vertrauen in eine Regierungsvertreterin, oder dasselbe wie ökonomisch qualifiziertes Vertrauen in ein bestimmtes Unternehmen, etc. Keines dieser Vertrauen ist per se grundlegender als andere Vertrauenstypen. Menschliches Leben in einer ausdifferenzierten Gesellschaft basiert auf ihnen allen bzw. bringt sie erst hervor. Vertrauen - als modales >Phänomen $<$ - ist eben nicht ursprünglich an diesen oder jenen, ökonomischen, politischen, moralischen, etc. Vertrauensverhältnissen zu fassen.

Abschliessend und zur nochmaligen Verdeutlichung: Politischen Verantwortungsträgerinnen und Vertretern der Wirtschaft ist nicht in erster Linie moralisches Vertrauen entgegenzubringen, wenn das bedeutet, dass wir diesen Menschen so begegnen, wie wir uns gegenüber Freunden, Lebenspartnern und anderen Personen, die uns

\footnotetext{
${ }^{46} \mathrm{Vgl}$. Annette Baier, Trust and Antitrust, in: Ethics 96, 2 (1986), 231-260, 235.

47 , Semiotisch qualifiziert, weil dieselbe Funktion auch von einem Wegweiser übernommen werden könnte.
} 
snah und lieb sind, verhalten (sollten). ${ }^{48}$ Verschiedene Vertrauenstypen haben vielmehr ihre eigenen normativen Rechtfertigungsbedingungen oder justification conditions $s{ }^{49}$ Daraus folgt natürlich nicht, dass wir zu (anderen) Politikerinnen und Wirtschaftsvertretern keine Freundschaftsbeziehungen eingehen sollten. Es ist vielmehr bloss gesagt, dass die empirischen und normativen Bedingungen, unter denen in einer typischen Situation Vertrauen gelingen oder misslingen, angebracht oder verfehlt sein kann, sachlich adäquat unterschieden sein wollen. Die Vergabe und Entgegennahme von Vertrauen verlangt nach kompetenten und verantwortlichen Subjekten, welche die normative Salienz einer vertrauensrelevanten Situation richtig einschätzen (lernen können). Grundlegend für eine solche Einschätzung ist die - meist intuitiv vorgenommene - Unterscheidung distinkter modaler Aspekte der Erfahrung bzw. deren typisches Zusammenspiel in einer konkreten Situation des Vertrauens-gebens und -nehmens. Die theoretische Nivellierung solcher Aspekte in Wissenschaft, Philosophie und Theologie widerspricht dem menschlichen Lebensvollzug in seiner irreduziblen Vielgestaltigkeit und ist daher letztlich zum Scheitern verurteilt.

\footnotetext{
${ }^{48}$ Mit dieser Verwendung des Ausdrucks 'moralisch folge ich einem anderen, enger gefassten Sprachgebrauch als dem oft üblichen, der ‘moralisch synonym mit sgut oder >richtig in einem vermeintlich transmodal-generischen Sinn verwendet.

49 Vgl. Karen Jones, Trust as an Affective Attitude, in: Ethics 107, 1 (1996), 4-25, 4.
} 


\section{Literatur}

Agamben, Giorgio. Herrschaft und Herrlichkeit. Zur theologischen Genealogie von Ökonomie und Regierung, Frankfurt a.M. 2010.

-, Homo Sacer. Il potere sovrano e la nuda vita,Torino 1995.

Baier, Annette, Trust and Antitrust, in: Ethics 96, 2 (1986) 231-260.

Berman, Harold J. Recht und Revolution. Die Bildung der westlichen Rechtstradition, Frankfurt a.M. 1991.

Brunner, Emil. Gerechtigkeit, Zürich 1943.

Chaplin, Jonathan, Herman Dooyeweerd. Christian Philosopher of State and Civil Society, Notre Dame 2011.

Cooper, Barry, The Political Theory of Eric Voegelin, Lewiston 1986.

Dahlstrom, Daniel O. Das logische Vorurteil. Untersuchungen zur Wahrheitstheorie des frühen Heidegger, Wien 1994.

Dooyeweerd, Herman, Die Philosophie der Gesetzesidee und ihre Bedeutung für die Rechts- und Sozialphilosophie, in: Archiv für Rechts- und Sozialphilosophie 53, 3; 4 (1967) 1-30; 465-513.

-, A New Critique of Theoretical Thought. 4 Bde., Lewiston 1997.

-, Reformation and Scholasticism in Philosophy. The Greek Prelude, Bd. 1, Lewiston 2004.

Feyerabend, Paul K., Die Vernichtung derVielfalt: Ein Bericht, Wien 2004.

Godfrey, Joseph J., Trust of People, Words, and God: A Route for Philosophy of Religion, Notre Dame 2012.

Habermas, Jürgen. Faktizität und Geltung. Beiträge zur Diskurstheorie des Rechts und des demokratischen Rechtsstaats 1, Frankfurt a.M. 1992.

Heidegger, Martin, Sein und Zeit. 19, Tübingen 2006.

Heisenberg, Werner, Der Teil und das Ganze. Gespräche im Umkreis der Atomphysik, München 1988.

Horkheimer, Max/Theodor Adorno, Dialektik der Aufklärung. Philosophische Fragmente 3, 2003.

Jandl, Martin J. Praxeologische Funktionalontologie. Eine Theorie des Wissens als Synthese von H. Dooyeweerd und R.B. Brandom. Frankfurt a.M. 2010.

Jones, Karen, Trust as an Affective Attitude, in: Ethics 107, 1 (1996) 4-25.

Kant, Immanuel, Kritik der praktischen Vernunft. Grundlegung zur Metaphysik der Sitten, hg. v. Wilhelm Weischedel, Zürich 1977.

Köchy, Kristian, Ganzheit und Wissenschaft: Das historische Fallbeispiel der romantischen Wissenschaft, Würzburg 1997.

Opitz, Peter Joachim/Gregor Sebba, The Philosophy of Order. Essays on History, Consciousness and Politics, Stuttgart 1981. 
Tillich, Paul, Liebe, Macht, Gerechtigkeit, übers. v. Gertie Siemsen u. Gertraut Stöber, Tübingen 1955.

Voegelin, Eric, Die kosmologischen Reiche des alten Orients - Mesopotamien und Ägypten, Bd. OG 1, München 2002.

-, Die Krise. Zur Pathologie des modernen Geistes, Paderborn 2007.

-, Die Neue Wissenschaft der Politik: Eine Einführung, München 1959.

-, Israel and Revelation, hg.v. Maurice P. Hogan, Bd. 14, Collected Works, Order \& History 1, Columbia 2001.

Williamson, Oliver E., Calculativeness, Trust, and Economic Organization, in: Journal of Law and Economics 36, 1 (1993) 453-486. Wolterstorff, Nicholas, Inquiring about God, Cambridge NY 2010.

Zuidervaart, Lambert, Artistic Truth. Aesthetics, Discourse, and Imaginative Disclosure, Cambridge UK 2004.

- Dr. Johannes Corrodi Katzenstein ist Privatdozent an der Theologischen Fakultät der Universität Zürich. 